\title{
DEVOLOPMENT OF MICROSTRUCTURE DAMAGE IN STRUCTURALLY HETEROGENEOUS MATERIALS UNDER DEFORMATION
}

\author{
V. V. Struzhanov ${ }^{1}$, S. S. Volkov ${ }^{1}$, T. A. Volkova ${ }^{2}$ \\ ${ }^{1}$ Institute of Engineering Science, Ural Branch of the Russian Academy of Sciences, 620049, 34 Komsomolskaya st., \\ Ekaterinburg, Russian Federation \\ ${ }^{2}$ Urals State University of Railway Transport, 620034, 66 Kolmogorova st., Ekaterinburg, Russian Federation
}

*Corresponding author. E-mail: volkovss48@yandex.ru; address for correspondence: ul. Komsomolskaya 34, 620049, Ekaterinburg, Russian Federation. Tel.:+7 (343) 37535 94; fax: +7 (343) 3745330

The proposed model represents a micro-heterogeneous medium with random properties of elastic-brittle microstructure elements and with fractures forming during the deformation of structurally heterogeneous materials. The probability of stress exceeding the ultimate strength in one element determines the probability of the failure of this element and the relative damage at the micro level. The suggested methodology for the calculation of damage is based on the use of a single parameter, specifically, the distribution density of the ultimate strengths of structural elements. Defining the increment step for the macro strain axis, we draw segments of the stress-strain curve taking into account the changed properties on each interval. The influence of damage on the stress-strain curve is observed. Uniaxial stress-strain diagram calculation for the exponential distribution of ultimate microstructure strengths in a model material is studied using the proposed methodology.

Keywords: random properties, microstructure damage, ultimate strength, stress-strain curve.

DOI: $10.17804 / 2410-9908.2016 .3 .021-030$

\section{References}

1. Sih G.C. Fracture mechanics in retrospect in contrast to multiscaling in prospect. In: Proceedings of the 17-th National Conference of Italian Group of Fracture, A. Finelli, L. Nobile, eds., Bologna, June 16-18, 2004, pp. 15-37.

2. Szuromi Ph. Microstructural Engineering of Materials. Science, 1997, vol. 277, no. 5330, pp. 1183. DOI: 10.1126/science.277.5330.1183.

3. Olson G.B. Computational Design of Hierarchically Structured Materials. Science, 1997, vol. 77, pp. 1237-1242. DOI: 10.1126/science.277.5330.1237.

4. Kornev V.M., Kurguzov V.D. Multiparametric sufficient criterion of quasi-brittle fracture for complicated stress state. Engineering Fracture Mechanics, 2008, vol. 75, iss. 5, pp. 1099-1113. DOI: 10.1016/j.engfracmech.2007.04.023.

5. Taylor D. The theory of critical distances. Engineering Fracture Mechanics, 2008, vol. 75, pp. 1696-1705. DOI: 10.1016/j.engfracmech.2007.04.007.

6. Trusov P.V., Volegov P.S., Yanz A.Yu. Two-Scale Models of Polycrystals: Evaluation of Validity of Ilyushin's Isotropy Postulate at Large Displacement Gradients. Physical Mesomechanics, vol. 19, iss. 1, pp. 21-34. DOI: 10.1134/S1029959916010033.

7. Volkov S.D., Stavrov V.P. Statisticheskaya mekhanika kompozitnykh materialov [Statistical Mechanics of Composite Materials]. Minsk, Belorus. Gos. Univ. Publ, 1978. (In Russian).

8. Volkov S.D. Statistical Strength Theory. Series: Russian Monographs and Texts on Advanced Mathematics and Physics, vol. XI. New York, Gordon and Breach, 1962.

9. Vildeman V.E., Sokolkin Yu.V., Tashkinov A.A. Mekhanika neuprugogo deformirovaniya $i$ razrusheniya kompozitsionnykh materialov [Mechanics of Non-Elastic Deformation and Fracture of Composite Materials]. Moscow, Nauka Publ., 1997. (In Russian). 
10. Volkova T.A., Volkov S.S. Microstructure damage related to deformation properties of grain composites. Theoretical and Applied Fracture Mechanics, 2008, vol. 49, iss. 3, pp. 242-250. DOI: 10.1016/j.tafmec.2008.02.004.

11. Volkova T.A., Volkov S.S. Microstructure damage related to stress- strain curve for grain composites. Theoretical and Applied Fracture Mechanics, 2009, vol. 52, iss. 2, pp. 83-90. DOI: 10.1016/j.tafmec.2009.08.007.

12. Zaitsev A.V. Second-order moment functions for the random structure of unidirectionally reinforced fibrous composites. In: Vestnik UGTU-UPI. Mekhanika microneodnorodnykh materialov i razrushenie [Herald of UGTU-UPI. Mechanics of Micro-Heterogeneous Materials and Fracture]. Ekaterinburg, GOU VPO UGTU-UPI Publ., 2006, no. 11 (82), pp. 161-167. (In Russian).

13. Surikova N.S., Panin V.E., Derevyagina L.S., Lutfullin R.Ya., Manzhina E.V., Kruglov A.A., Sarkeeva A.A. Micromechanisms of Deformation and Fracture in a VT6 Titanium Laminate under Impact Load. Phys. Mesomech., 2015, vol. 18, iss. 3, pp. 250-260. DOI: $10.1134 / \mathrm{S} 1029959915030091$.

14. Schastlivtsev V.M., Tabatchikova T.I., Yakovleva I.L., Klyueva S.Yu., Kruglova A.A., Khlusova E.I., Orlov V.V. Microstructure and properties of low-carbon weld steel after thermomechanical strengthening. The Physics of Metals and Metallography, 2012, vol. 113, iss. 5, pp. 480-488. DOI: 10.1134/S0031918X12050067.

15. Yokobori T. An Interdisciplinary Approach to Fracture and Strength of Solids. Groningen, Wolters-Noordhoff Scientific Ltd, 1968.

16. Tamuzs V.P., Kuksenko V.S. Micromekhanika rasrushenia polimernykh materialov [Micromechanics of Fracture of Polymeric Materials]. Riga, Zinatne Publ., 1978, 296 p. (In Russian).

17. Struzhanov V.V., Bashurov V.V., Tartashnik K.A. On one approach to the modeling of elastic-brittle material properties. In: Vestnik UGTU-UPI. Mekhanika microneodnorodnykh materialov $i$ razrushenie [Herald of UGTU-UPI. Mechanics of Micro-Heterogeneous Materials and Fracture]. Ekaterinburg, GOU VPO UGTU-UPI Publ., 2004, no. 22 (52), pp. 99-109. (In Russian).

18. Privalova V.V., Struzhanov V.V. Some regularities in the behavior of elastic-brittle material under cyclic tension. Uchenye zapiski Komsomolskogo-na-Amure gosudarstvennogo tekhnicheskogo universiteta. Ser. Nauki o prirode i tekhnike, 2012, no. I-1 (9), pp. 94-99. (In Russian).

19. Encyclopedia of Physics. Flügge S., ed. Mechanics of Solids I, vol. VI a/1, S. Truesdell, ed. Berlin-Heidelberg-New York, Springer-Verlag, 1973.

20. Fridman Ya.B. Mekhanicheskie svoistva metallov [Mechanical Properties of Metals. Part 1. Deformation and Fracture]. M., Mashinostroenie Publ., 1974, 472 p. (In Russian).

21. Volkov S.S. Mekhanika anizotropnykh kompozitov [Mechanics of Anisotropic Composites]. Ekaterinburg, UrO RAN Publ., 2010, 85 p. (In Russian). 
Подана в журнал: 17.04 .2016

УДК 539.3

DOI: $10.17804 / 2410-9908.2016 .3 .021-030$

\title{
РАЗВИТИЕ МИКРОСТРУКТУРНОЙ ПОВРЕЖДЕННОСТИ ПРИ ДЕФОРМИРОВАНИИ СТРУКТУРНО-НЕОДНОРОДНЫХ МАТЕРИАЛОВ
}

\author{
В. В. Стружанов ${ }^{1}$, С. С. Волков ${ }^{1}$, Т. А. Волкова ${ }^{2}$ \\ ${ }^{1}$ Федеральное государственное бюджетное учреждение науки Институт машиноведения Уральского \\ отделения Российской академии наук, ул. Комсомольская, 34, Екатеринбург, Российская Федераиия \\ ${ }^{2}$ Федеральное государственное бюджетное образовательное учреждение высшего профессионального \\ образования «Уральский государственный университет путей сообщения», ул. Колмогорова, 66, Екатеринбург, \\ Российская Федераичи \\ *Ответственный автор. Электронная почта: volkovss48@yandex.ru; адрес для переписки: ул. Комсомольская 34, \\ Екатеринбург, Российская Федерация; телефон: +7 (343) 375-35-94; факс: +7 (343) 374-53-30
}

Предложена модель микронеоднородной среды со случайными свойствами элементов микроструктуры и поврежденностью, возникающей при деформировании структурнонеоднородных материалов. Поврежденность микроструктуры рассчитывается как вероятность превышения в элементах микроструктуры случайного напряжения над случайным пределом прочности. Приведена методика расчета поврежденности, основанная на использовании одного параметра, а именно, плотности распределения пределов прочности структурных элементов. Расчет поврежденности дает возможность на каждом шаге нагружения находить макроскопические свойства композита и строить соответствующие участки диаграммы деформирования. Показано влияние поврежденности на диаграмму деформирования. В качестве примера применения методики выполнены расчеты диаграммы одноосного растяжения для экспоненциального закона распределения пределов прочности микроструктуры модельного материала.

Ключевые слова: случайные свойства, поврежденность микроструктуры, предел прочности, диаграммы деформирования.

\section{1. Введение}

Значительный интерес в настоящее время проявляется к разработке многоуровневых моделей конструкционных материалов [1-6]. Изучение влияния свойств микроструктуры металлов проводится на моделях, учитывающих микро- и макросвойства деформируемой среды. Металлы рассматриваются как частный случай стохастических композиционных материалов [7-11]. Элементы на макроуровне обладают постоянными детерминированными свойствами. Элементы микроструктуры - это элементы второго порядка малости. Они обладают случайными деформационными и прочностными свойствами.

Методы прогнозирования деформационных свойств композитов различной структуры достаточно хорошо разработаны $[7,9,10]$. Для анализа неоднородности микроструктуры композита используются методы теории вероятностей. В большинстве работ при этом рассматриваются двухкомпонентные композиты с дискретными законами распределения. При этом применение непрерывных распределений предоставляет значительно больше возможностей для исследования [10]. Данные экспериментов позволяют найти параметры распределения случайных свойств микроструктуры материала [7, 12]. При этом изучаются микрошлифы металлов, распределение легирующих элементов, анализируется наличие микрополостей и микротрещин $[13,14]$. Используются методы металлографии и электронной микроскопии. 
В процессе эксплуатации развивается поврежденность элементов микроструктуры. Многомасштабные критерии разрушения были получены для хрупких сред в работах $[4,5]$. На ранних стадиях разрушение имеет рассеянный, дисперсный характер [15-17]. Свойства микроструктуры при этом можно считать статистически независимыми. Относительное число разрушенных элементов микроструктуры находится с помощью микроструктурного условия прочности. Это условие зависит от вида нагружения материала и включает параметры распределения случайных микроструктурных напряжений $[11,18]$.

Накопление поврежденности в ходе нагружения влияет на связь между напряжениями и деформациями и приводит к постепенной деградации свойств материала. На начальной стадии упругого деформирования в микроструктуре возникает незначительная поврежденность $[9,19,20]$, которой, как правило, пренебрегают. Дальнейшее развитие поврежденности уже оказывает существенное влияние, что отражается нелинейной зависимостью напряжений и деформаций. Таким образом, микроструктура и её поврежденность определяют свойства структурно-неоднородных материалов и, в конечном итоге, эксплуатационные возможности элементов конструкций.

В данной работе сделана попытка, опираясь на некоторые известные положения механики структурно-неоднородных материалов, а также на полученные оригинальные результаты, построить методику расчета поврежденности микроструктуры и на этой базе создать алгоритм прогнозирования диаграммы связи напряжений и деформаций, отражающей упругую и неупругую стадии деформирования. Особенность исследования заключается в том, что используется только один параметр, а именно, плотность распределения пределов прочности элементов микроструктуры.

\section{2. Поврежденность микроструктуры материалов}

В исследовании используется математическая модель микронеоднородной среды, содержащей элементы одного типа - упруго-хрупкие. Рассмотрим элементарный макрообъем материала, состоящий из множества элементов микроструктуры, размеры которых существенно меньше размера данного макрообъема.

Свойства элементов микроструктуры имеют случайный характер, и, следовательно, их пределы прочности определяются случайной величиной $S(X)$, где $X$ - точка микрообъема. Микроструктурный предел прочности $S(X)$ определяется в зависимости от вида напряженного состояния. В общем случае рассматривается тензорная форма записи напряжений [11]. В различных теориях прочности понятие предела прочности определяется как предельно допустимое значение некоторой функции компонентов тензора напряжений. Для такой функции обычно используется термин эквивалентное напряжение. Для произвольного напряженного состояния случайную величину $S(X)$ представим как предельно допустимое значение эквивалентных напряжений в элементах микроструктуры.

Пусть в результате воздействия внешних усилий в микрообъеме возникло случайное поле напряжений, характеризуемое случайным эквивалентным напряжением $\sigma(X)$. Тогда микроструктурное условие прочности можно представить как разницу между случайным эквивалентным напряжением $\sigma(X)$ и случайным пределом прочности $S(X)$ :

$$
w(X)=\sigma(X)-S(X) .
$$

Если параметр $w(X)>0$, то напряжение в точке $X$ больше предела прочности, поэтому в элементе микроструктуры произойдет разрушение. При $w(X)<0$ разрушение не происходит, так как напряжение находится в допустимой области безопасных значений.

Множество реализаций случайной величины ограничено (в силу естественных причин $0<S(X)<\infty, 0<\sigma(X)<\infty)$ и обладает мощностью континуума. Поэтому эти реализации полностью заполняют некоторый отрезок $[a, b]$ на действительной оси $0 x$, причем 
$a \leq 0 \leq b$. Пусть известна плотность распределения $f_{w}(x)$ непрерывной случайной величины $w$, тогда поврежденность микроструктуры $q$ (объемное содержание разрушенных элементов микроструктуры) равна

$$
q=\int_{0}^{\infty} f_{w}(x) d x
$$

Упростим задачу, полагая, что реализовано однородное (неслучайное) поле напряжений с эквивалентным детерминированным напряжением $\sigma$. Тогда в элементе микроструктуры произойдет разрушение, если $w(X)=\sigma-S(X)>0$. Реализации непрерывной случайной величины $S$ расположены в отрезке $\left[S_{1}, S_{2}\right]$, где $S_{1}$ - прочность самого слабого элемента микроструктуры; $S_{2}$ - наиболее крепкого $\left(S_{1}>0, S_{2}>0\right)$. Если на этом отрезке задана плотность распределения случайной величины $S$, которая естественно совпадает по своим характеристикам с плотностью случайной величины $w$, то поврежденность вычисляется по формуле

$$
q=\int_{S_{1}}^{\sigma} f(x) d x
$$

являющейся результатом преобразования формулы (1).

Приведем расчет поврежденности, предполагая, что случайная величина $S$ распределена по закону, близкому к экспоненциальному с плотностью распределения:

$$
f(x)=\left\{\begin{array}{l}
0 \quad \text { nри } x<S_{1} \\
\frac{1}{S} \exp \left(-\frac{x-S_{1}}{S}\right) \quad \text { при } x \geq<S_{1},
\end{array}\right.
$$

где $S=\langle S(X)>-$ математическое ожидание пределов прочности. Тогда

$$
q=\int_{S_{I}}^{\sigma} f(x) d x=1-\exp \left(-\frac{\sigma-S_{1}}{S}\right) .
$$

На рис. 1 показан график плотности распределения предела прочности зерен микроструктуры. Площадь заштрихованной области над интервалом $\left[S_{1}, \sigma\right]$ равна относительной микроструктурной поврежденности.

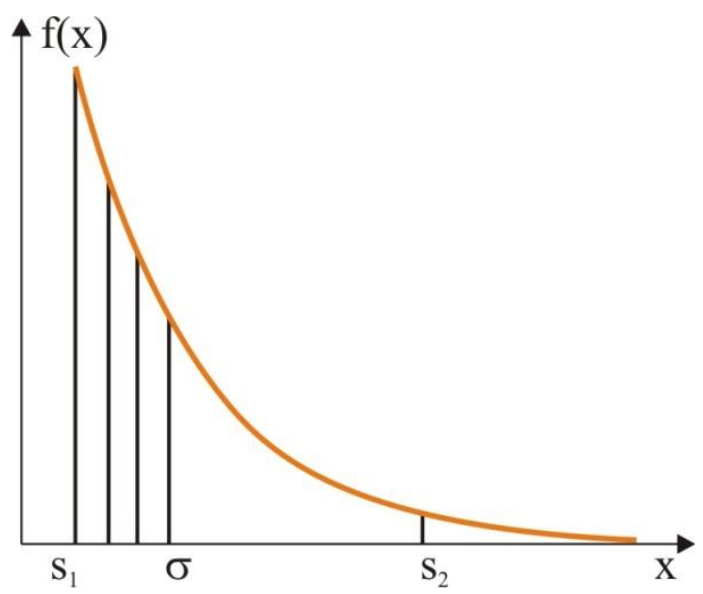

Рис. 1. Плотность распределения микроструктурного предела прочности 


\section{3. Макромодули поврежденного материала}

Модули микроструктуры являются случайными величинами. Это $K(X)$ - модуль объемной деформации; $G(X)$ - модуль сдвиговой деформации; $E(X)$ - модуль Юнга. Полагаем, что коэффициент Пуассона $v$ - детерминированная величина, одинаковая для любого элемента микроструктуры. Математические ожидания модулей: $E=\langle E(X)\rangle, K=\langle K(\mathrm{X})\rangle$, $G=\langle G(X)\rangle$. Тензор случайных модулей упругости $\Theta(X)$ выражается через случайные модули $K(X)$ и $G(X)$ следующим образом[9, 18$]$ :

$$
\Theta(X)=3 K(X) \mathrm{V}+2 G(X) \mathrm{D},
$$

где V - объемная, D - девиаторная составляющие единичного тензора четвертого ранга I.

При расчете макроскопических свойств материала к тензору средних модулей упругости $\mathrm{C}=\langle\Theta(X)>$ добавляется тензор поправок $\mathrm{h}[6,9,18]$.

$$
\mathrm{h}=3 \Delta K \cdot \mathrm{V}+2 \Delta G \cdot \mathrm{D}
$$

где $\Delta K$ и $\Delta G$ поправки к средним модулям объемной $\Delta K$ и сдвиговой $G$ деформации. В результате получаем тензор эффективных модулей упругости $\tilde{C}=\mathrm{C}+\mathrm{h}$.

Для пористых материалов, в которых часть микроэлементов разрушена, возможно ввести случайную индикаторную функцию $\lambda(X)$, равную единице для неразрушенного микроэлемента и нулю для разрушенного. Тогда $p=\langle\lambda(X)\rangle-$ относительное содержание целых подэлементов, а $q=1-p-$ поврежденность (относительное содержание разрушенных подэлементов). В работе [9] для таких материалов получены следующие выражения:

$$
\Delta \mathrm{K}=-\mathrm{pqK} \sum_{\mathrm{n}=1}^{\infty}\left(\mathrm{p}^{\mathrm{n}}-(-\mathrm{q})^{\mathrm{n}}\right) \mathrm{d}^{\mathrm{n}} ; \quad \Delta \mathrm{G}=-\mathrm{pqG} \sum_{\mathrm{n}=1}^{\infty}\left(\mathrm{p}^{\mathrm{n}}-(-\mathrm{q})^{\mathrm{n}}\right) \mathrm{t}^{\mathrm{n}} \cdot d=\frac{3 K}{3 K+4 G}, \quad t=\frac{6(K+2 G)}{5(3 K+4 G)}
$$

Полученные ряды являются геометрическими прогрессиями. Для их сходимости достаточно условия $\max \{p d, q d, p t, q t\}<1$. Суммируя прогрессии, получим

$$
\Delta K=-K \frac{p q d}{(1-p d)(1+q d)} ; \quad \Delta G=-G \frac{p q t}{(1-p t)(1+q t)}
$$

Зная поправки $\Delta K, \Delta G$, вычислим макромодули (эффективные модули) упругости материала $\tilde{K}=K+\Delta K, \widetilde{G}=G+\Delta G$ :

$$
\tilde{K}=p K\left(1-\frac{q d}{(p+q d)(1-d)}\right) ; \quad \tilde{G}=p G\left(1-\frac{q t}{(p+q t)(1-t)}\right)
$$

Этот же результат можно выразить через средний модуль Юнга и коэффициент Пуассона:

$$
\begin{array}{ll}
\tilde{K}=\frac{p E}{3(1-2 v)}\left(1-\frac{q d}{(p+q d)(1-d)}\right), & d=\frac{1+v}{3(1-v)}, \\
\tilde{G}=\frac{p E}{2(1+v)}\left(1-\frac{q t}{(p+q t)(1-t)}\right), & t=\frac{8-10 v}{15(1-v)} .
\end{array}
$$


Пользуясь известными формулами [20], найдем макромодули (эффективные модули) $\widetilde{E}, \tilde{v}:$

$$
\tilde{E}=\frac{9 \tilde{K} \tilde{G}}{3 \tilde{K}+\tilde{G}} ; \quad \tilde{v}=\frac{3 \tilde{K}-2 \tilde{G}}{6 \tilde{K}+2 \tilde{G}}
$$

На рис. 2 представлена зависимость макромодуля Юнга $\tilde{E}$ от поврежденности $q$ при различных значениях коэффициента Пуассона v. Начальный модуль Юнга сплошного материала принят равным единице. Чем больше начальный коэффициент Пуассона, тем быстрее накапливаются изменения от $q$. С приближением критической поврежденности макромодуль $\widetilde{E}$ переходит в область отрицательных значений.

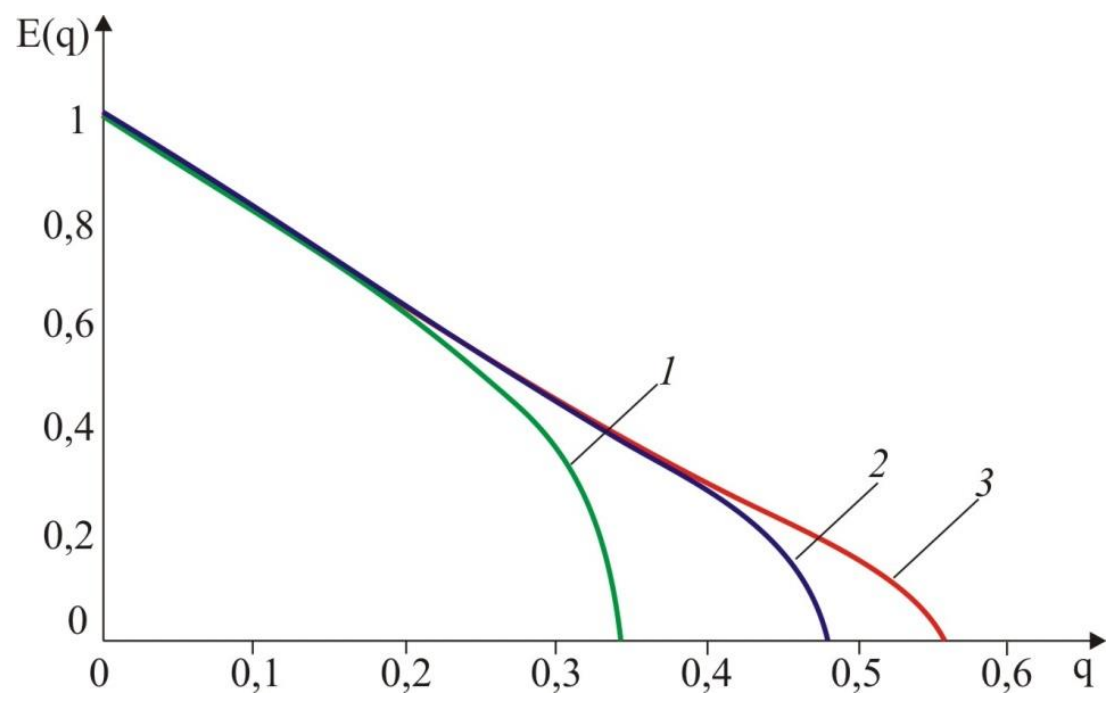

Рис. 2. Зависимость макромодуля Юнга $E$ от поврежденности микроструктуры $q$ при различных коэффициентах Пуассона: $1-v=0,35 ; 2-v=0,31 ; 3-v=0,27$

\section{4. Поврежденность микроструктуры и диаграмма деформирования при одноосном растяжении}

Осуществим теперь одноосное растяжение структурно-неоднородного материала с начальным макромодулем Юнга $E$ и коэффициентом Пуассона v. Случайный предел прочности $S(X)$ представляет собой случайное временное сопротивление разрыва для одноосного растяжения с математическим ожиданием (средним значением) $S$. Деформирование производим поэтапно по жесткой схеме, задавая однородную по объему макродеформацию растяжения $e=e_{i}=i \Delta e(i=1,2, \ldots n, \Delta e-\mathrm{const})$. Тогда на каждом (i+1)-м этапе макронапряжение определяется выражением

$$
\sigma=\sigma_{i+1}=\sigma_{i}+\tilde{E}_{i}\left(q_{i}\right) \cdot \Delta e
$$

Здесь $\tilde{E}_{i}-$ макромодуль, вычисленный для поврежденности $q_{i}$, достигнутой на предыдущем этапе.

Таким образом, зависимость напряжения от деформации можно рассчитать, используя следующий алгоритм. Пусть на $i$-м шаге известны величины $\sigma_{i}, e_{i}, \tilde{E}_{i}$. Производя догружение по формуле (5), находим $\sigma_{i+1}$. Затем по формуле (2), где $\sigma=\sigma_{i+1}$ для известной плотности распределения пределов прочности микроэлементов вычисляем поврежденность $q_{i+1}$. Нако- 
нец, по формулам $(3,4)$ определяем новое значение макромодуля $\tilde{E}_{i+1}$. После этого осуществляем следующее догружение и так далее.

На рис. 3 показаны результаты расчетов поврежденности материала при $E=100$ ГПа, $v=0,31, \Delta e=0,002$. Пусть $S_{1}=80 \mathrm{MПа-} \mathrm{прочность} \mathrm{самого} \mathrm{слабого} \mathrm{элемента} \mathrm{микрострукту-}$ ры. Пределы прочности микроструктуры подчиняются экспоненциальному закону распределения, заданному выше. Рассмотрим три варианта значений математического ожидания предела прочности: $1-S=250 \mathrm{MПа;} 2-S=300 \mathrm{MПа;} 3-S=400$ МПа. Отметим, что быстрее всего развивается поврежденность для материала с низким уровнем математического ожидания прочности, что соответствует кривой 1 на рис. 3.

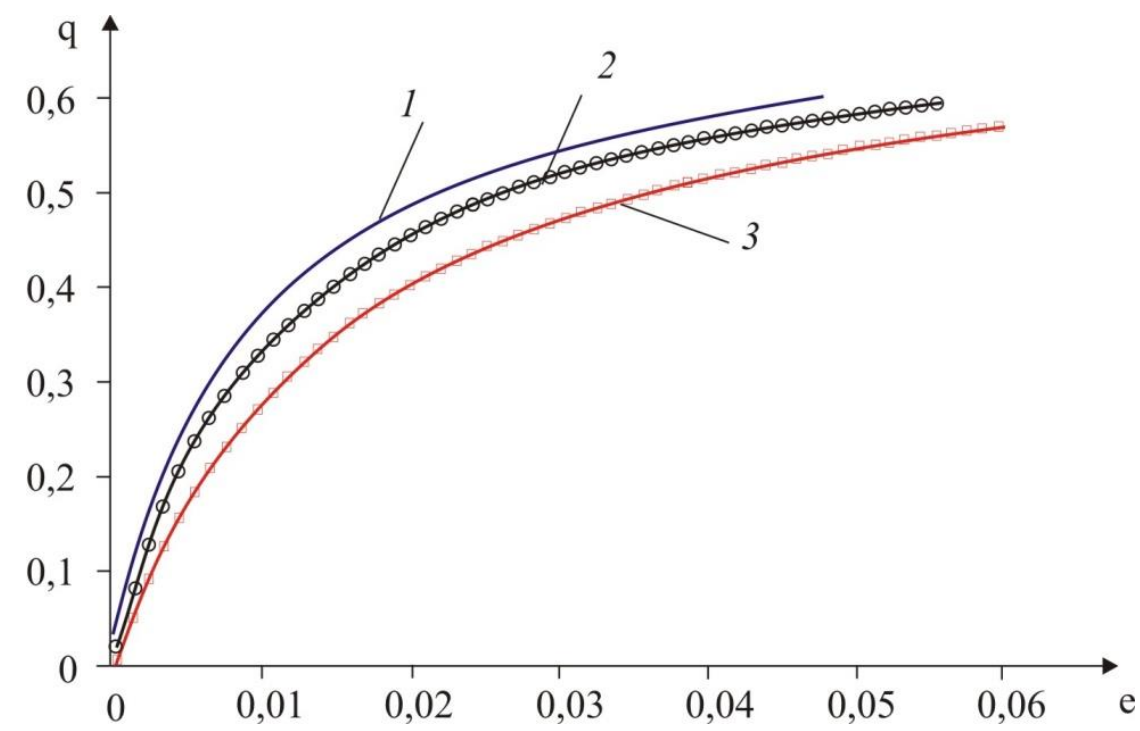

Рис. 3. Зависимость поврежденности от величины деформации

при различных параметрах распределения пределов прочности микроструктуры:

$1-S=250 \mathrm{M \Pi а} ; 2-S=300 \mathrm{M \Pi а} ; 3-S=400 \mathrm{MПа}$

На рис. 4 представлены зависимости между напряжениями и деформациями для тех же самых параметров.

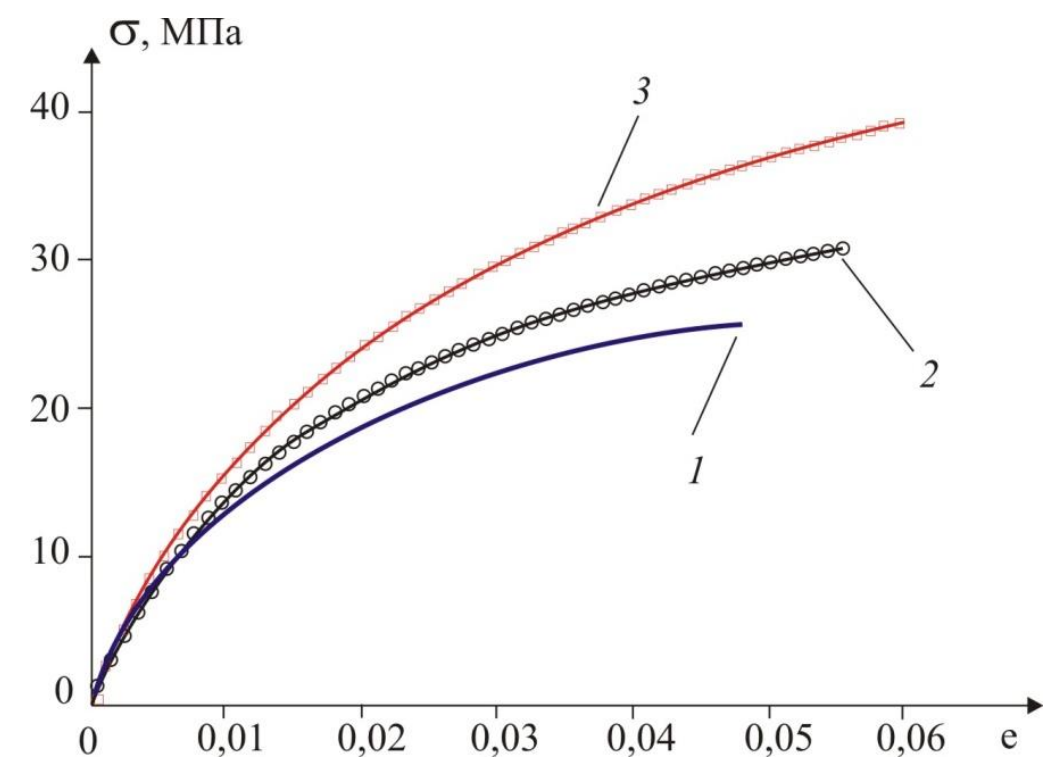

Рис. 4. Диаграммы деформирования при различных параметрах распределения пределов прочности микроструктуры:

$$
1-S=250 \text { МПа; } 2-S=300 \text { МПа; } 3-S=400 \text { Мпа }
$$




\section{5. Заключение}

Разработана методика расчета поврежденности материала, опирающаяся на использование плотности распределения пределов прочности его микроструктурных элементов. Приведен алгоритм прогнозирования диаграммы деформирования материала по заданной плотности распределения пределов прочности элементов микроструктуры. Данные результаты открывают возможность проектирования материалов с наперед заданными свойствами при варьировании свойств микроструктуры. Также, используя изложенную теорию, можно выйти на решение обратной задачи - определения параметров микроструктуры по экспериментально построенной макродиаграмме деформирования.

\section{Литература}

1. Sih G. C. Fracture mechanics in retrospect in contrast to multiscaling in prospect // The 17-th National Conference of Italian Group of Fracture, Bologna, June 16-18, 2004 : proceedings / by education of A. Finelli, L. Nobile. - P. 15-37.

2. Szuromi Ph. Microstructural Engineering of Materials // Science. - 1997. - Vol. 277, no. 5330. - P. 1183. - DOI: 10.1126/science.277.5330.1183.

3. Olson G. B. Computational Design of Hierarchically Structured Materials // Science. 1997. - Vol. 77. - P. 1237-1242. - DOI: 10.1126/science.277.5330.1237.

4. Kornev V. M., Kurguzov V. D. Multiparametric sufficient criterion of quasi-brittle fracture for complicated stress state // Engineering Fracture Mechanics. - 2008. - Vol. 75, iss. 5. P. 1099-1113. - DOI: 10.1016/j.engfracmech.2007.04.023.

5. Taylor D. The theory of critical distances // Engineering Fracture Mechanics. - 2008. Vol. 75. - P. 16961705. - DOI: 10.1016/j.engfracmech.2007.04.007.

6. Trusov P. V., Volegov P. S., Yanz A. Yu. Two-Scale Models of Polycrystals: Evaluation of Validity of Ilyushin's Isotropy Postulate at Large Displacement Gradients // Physical Mesomechanics. - Vol. 19, iss. 1. - P. 21-34. - DOI: 10.1134/S1029959916010033.

7. Волков С. Д., Ставров В. П. Статистическая механика композитных материалов. Минск : БГУ, 1978. - 206 с.

8. Volkov S. D. Statistical Strength Theory. Series: Russian Monographs and Texts on Advanced Mathematics and Physics. Vol. XI. - New York : Gordon and Breach, 1962.

9. Вильдеман В. Э., Соколкин Ю. В., Ташкинов А. А. Механика неупругого деформирования и разрушения композитных материалов / под ред. Ю. В. Соколкина. - М. : Наука. Физматлит, 1997. - 288 с.

10. Volkova T. A., Volkov S. S. Microstructure damage related to deformation properties of grain composites // Theoretical and Applied Fracture Mechanics. - 2008 - Vol. 49, iss. 3. P. 242-250. - DOI: 10.1016/j.tafmec.2008.02.004.

11. Volkova T. A., Volkov S. S. Microstructure damage related to stress- strain curve for grain composites // Theoretical and Applied Fracture Mechanics. - 2009. - Vol. 52, iss. 2. - P. 83-90. DOI: 10.1016/j.tafmec.2009.08.007.

12. Зайцев А. В. Моментные функции второго порядка случайной структуры однонаправленно армированных волокнистых композитов // Вестник УГТУ-УПИ. Механика микронеоднородных материалов и разрушение : сборник научных трудов. - Екатеринбург : УГТУ-УПИ, 2006. - № 11 (82). - С. 161-167.

13. Micromechanisms of Deformation and Fracture in a VT6 Titanium Laminate under Impact Load / N. S. Surikova, V. E. Panin, L. S. Derevyagina, R. Ya. Lutfullin, E. V. Manzhina, A. A. Kruglov, A. A. Sarkeeva // Physical Mesomechanics. - 2015. - Vol. 18, iss. 3. - P. 250-260. DOI: $10.1134 / \mathrm{S} 1029959915030091$.

14. Microstructure and properties of low-carbon weld steel after thermomechanical strengthening / V. M. Schastlivtsev, T. I. Tabatchikova, I. L. Yakovleva, S. Yu. Klyueva, A. A. Kruglova, 
E. I. Khlusova, V. V. Orlov // The Physics of Metals and Metallography. - 2012. - Vol. 113, iss. 5. P. 480-488. - DOI: 10.1134/S0031918X12050067.

15. Yokobori T. An Interdisciplinary Approach to Fracture and Strength of Solids. Groningen : Wolters-Noordhoff Scientific Ltd, 1968.

16. Тамуж В. П., Куксенко В. С. Микромеханика разрушения полимерных материалов. Рига : Зинатне, 1978. - 296 с.

17. Стружанов В. В., Башуров В. В., Тарташник К. А. Об одном подходе к моделированию свойств упругохрупкого материала // Вестник УГТУ-УПИ. Механика микронеоднородных материалов и разрушение : сборник научных трудов. - Екатеринбург : ГОУ ВПО УГТУ-УПИ, 2004. - № 22 (52). - С. 99-109.

18. Привалова В. В., Стружанов В. В. Некоторые закономерности изменения свойств упругохрупкого материала в ходе циклического растяжения // Ученые записки Комсомольского-на Амуре государственного технического университета. Сер. Науки о природе и технике. - 2012. - № I-1 (9). - C. 94-99.

19. Encyclopedia of Physics / by education of S. Flügge. Vol. VI a/1. Mechanics of Solids I / by education of S. Truesdell. - Berlin-Heidelberg-New York : Springer-Verlag, 1973.

20. Фридман Я. Б. Механические свойства металлов. Часть 1. Деформация и разрушение. - М. : Машиностроение, 1974. - 472 с.

21. Волков С. С. Механика анизотропных композитов. - Екатеринбург : УрО РАН, 2010. $85 \mathrm{c}$. 